\title{
Observation of Electronic Structure in Conduction Band of $\mathrm{CaTiO}_{3}$
}

\author{
Kazushige UEDA, Hiroshi YANAGI, Ryuichi NOSHIRO, Hiroshi MIZOGUCHI*, Takahisa OMATA**, \\ Naoyuki UEDA ${ }^{\dagger}$, Hideo HOSONO and Hiroshi KAWAZOE \\ Materials and Structures Laboratory, Tokyo Institute of Technology, 4259, Nagatsuta, Midori-ku, Yokohama-shi 226-8503 \\ * Osaka National Research Institute, 1-8-31, Midorigaoka, Ikeda-shi, Osaka 563-8577 \\ ** Graduate School of Engineerng, Osaka University, 2-1, Yamadaoka, Suita-shi, Osaka 565-0871
}

\author{
$\mathrm{CaTiO}_{3}$ の伝導帯電子構造の観察 \\ 植田和茂・柳＼cjkstart博・能代龍一・溝口 拓* $\cdot$ 小俣孝久 ${ }^{* *} \cdot$ 植田尚之 $\cdot$ 細野秀雄・川副博司 \\ 東京工業大学応用セラミックス研究所, 226-8503 横浜市緑区長津田町 4259 \\ *大阪工業技術研究所, 563-8577 池田市緑丘 1-8-31 \\ **大阪大学大学院工学研究科, 565-0871 吹田市山田丘 2-1
}

\begin{abstract}
Electronic structure in the conduction band of $\mathrm{CaTiO}_{3}$ was examined by the bremsstrahlung isochromat spectroscopy (BIS), X-ray absorption spectroscopy (XAS) and electron energy loss spectroscopy (EELS). Two broad bands were observed at 2 and $7 \mathrm{eV}$ above the Fermi energy in the BIS spectrum. The features of the XAS spectra for $\mathrm{Ca} \mathrm{L} \mathrm{L}_{2,3}$, Ti $\mathrm{L}_{2,3}$, and $\mathrm{O} \mathrm{K}$ edges were almost the same as those of the EELS spectra, and the XAS spectrum for each element was in reasonable agreement with its partial density of states estimated by an energy band calculation. A trial spectrum was synthesized by the sum of the XAS spectra, and it was found that the feature of the synthesized spectrum is similar to that of the BIS spectrum. From the comparison between XAS and BIS spectra, the two broad bands at 2 and $7 \mathrm{eV}$ in the BIS spectrum were assigned to $\mathrm{Ti} 3 \mathrm{~d}$ bands and $\mathrm{Ca} 4 \mathrm{~s}$ bands, respectively.

[Received March 17, 1998; Accepted July 13, 1998]
\end{abstract}

Key-words : $\mathrm{CaTiO}_{3}$, Electronic structure, Conduction band, Photoemission spectra, BIS spectra, EELS spectra

\section{Introduction}

$\mathrm{CaTiO}_{3}$ is involved in a family of alkaline earth titanates with perovskite structure. The crystal structure of $\mathrm{CaTiO}_{3}$ is not an ideal cubic perovskite type but a $\mathrm{GdFeO}_{3}$ type with orthorhombic distortion, ${ }^{1)}$ because the ionic radius of a $\mathrm{Ca}$ ion is smaller than that of a $\mathrm{Sr}$ or $\mathrm{Ba}$ ion. Alkaline earth titanates generally show $n$-type electrical conduction by proper donor doping, and have some useful properties for electrical and optical applications. Extensive and intensive studies on their electronic structures have been undertaken to find the origins of their electrical properties, especially for $\mathrm{SrTiO}_{3}{ }^{2)-11)}$ and $\mathrm{BaTiO}_{3}{ }^{12)-14)}$ On the other hand, only a few studies have been reported in $\mathrm{CaTiO}_{3},{ }^{15)}$ which is probably due to less interest in practical applications and its complicated crystal structure. We have studied the electroconductive properties of non-doped and doped $\mathrm{CaTiO}_{3}$ single crystals, and their electrical conductivity was examined. ${ }^{16)}$ Analysis of the electronic structure of $\mathrm{CaTiO}_{3}$ will provide useful information about the conductivity, and it will be meaningful for the total understanding of the electroconductive properties.

Most of oxide semiconductors such as $\mathrm{In}_{2} \mathrm{O}_{3}, \mathrm{SnO}_{2}$ and $\mathrm{TiO}_{2}$ show $n$-type electrical conduction reflecting their electronic structure. Although some exceptions have been reported, ${ }^{17)}$ the bottom of the conduction bands of the oxide semiconductors mainly consists of $n \mathrm{~s}^{0}$ or $n \mathrm{~d}^{0}$ orbitals of cations, and the valence bands are composed of $2 \mathrm{p}$ orbitals of oxygen ions. Since the dispersion of the conduction bands is usually much larger than that of the valence bands, the mobility of electrons is considerably larger than that of positive holes, which results in the $n$-type electrical conduction observed in the oxide semiconductors. Direct observation of the conduction bands is requested for the analysis of

\footnotetext{
${ }^{\dagger}$ Now with Research Center, Sony Corporation, 174, Fujitsukacho, Hodogaya-ku, Yokohama-shi 240-0031

現在：ソニ一(株)中央研究所, 240-0031 横浜市保土ケ谷区藤 塚町 174
}

the electroconductive properties because the conduction bands play an important role in the transport of electrons. Therefore, for the examination of the conduction bands, inverse photoemission spectroscopy (IPES), X-ray absorption spectroscopy (XAS) and electron energy loss spectroscopy (EELS) are more useful than photoemission spectroscopy (PES) which observes occupied bands. IPES generally gives the total density of states (DOS) in the conduction bands of materials, while XAS and EELS do the partial DOS (PDOS) of each element constituting the materials. A combination of these spectroscopy will tell us some invaluable images of electronic structures in the conduction bands.

Information about the conduction bands of oxide semiconductors is useful for understanding of their various optical properties as well as electronic transport properties. Some titanates are known as photoconductive, photovoltaic or photocatalytic materials. In these materials, final states on optical transitions will influence the magnitude of photocurrent or photovoltage, selectivity in the catalytic reaction, and quantum yield of the optical transitions. Moreover, various luminescence phenomena and nonlinear optical phenomena under densely photo-excited states are observed in some oxides such as $\mathrm{BaTiO}_{3}$. The virtual or higher excited states causing the phenomena under the excitation will be closely related to unoccupied states of the materials. Understanding of electronic structure in the conduction bands of materials has been increasingly important to interpret the origins of the properties and develop new materials with novel optical functions.

In the present study, electronic structure in the conduction band of $\mathrm{CaTiO}_{3}$ was examined by some spectroscopic techniques. The total DOS in the conduction band was observed directly by bremsstrahlung isochromat spectroscopy (BIS), which belongs to IPES techniques. The PDOSs derived from the orbitals of each ion were observed by XAS and EELS measurements, and each spectrum was compared with the corresponding PDOS estimated by a 
tight-binding energy band calculation. In addition, a trial spectrum was synthesized by the sum of the XAS spectra to simulate the total DOS, and the spectrum synthesized was compared with the BIS spectrum.

\section{Experimental}

Single crystals of $\mathrm{CaTiO}_{3}$ were prepared by the floating zone method using an infrared furnace. ${ }^{16)}$ Nominally nondoped crystals were used in the EELS measurement, whereas electroconductive crystals doped with Y 0.01 at\% $\left(\mathrm{CaTi}_{0.9999} \mathrm{Nb}_{0.0001} \mathrm{O}_{3}\right)$ were used in the BIS and XAS experiments to avoid charging effects. Since the major chemical components in the crystals are responsible for the results of the measurements, effects of Y-doping on the spectra were assumed to be negligible.

BIS measurement was carried out using an instrument built up in our laboratory, which detects $9.4 \mathrm{eV}$ photons by an electron multiplier (Hamamatsu Photonics: R595) through a $\mathrm{SrF}_{2}$ window. The BIS is called as UV-BIS in the present paper because the light in the ultraviolet (UV) region is detected. The surface of a sample was scraped by a diamond file at a pressure of $\sim 10^{-7} \mathrm{~Pa}$ in the preparation chamber, and the sample was promptly transferred into the analysis chamber. BIS spectra were measured under the vacuum level of $\sim 10^{-8} \mathrm{~Pa}$. The energy resolution of the BIS spectra was $\sim 1 \mathrm{eV}$ and the energy scale was calibrated by the Fermi energy of Au measured under the same conditions.

XAS measurements for $\mathrm{Ca}_{2,3}$ edges, $\mathrm{Ti}_{2,3}$ edges and $\mathrm{O}$ $\mathrm{K}$ edge were performed in a total photoelectron yield mode on BL2B1 of UVSOR at Institute for Molecular Science (IMS). The surface of a sample was scraped by a diamond file at a pressure of $\sim 10^{-7} \mathrm{~Pa}$ before the measurements and the spectra were obtained under a vacuum of $\sim 10^{-8} \mathrm{~Pa}$. The energy resolution was less than $1 \mathrm{eV}$.

EELS spectra for $\mathrm{CaL}_{2,3}$ edges, $\mathrm{TiL}_{2,3}$ edges and $\mathrm{OK}$ edge were measured using a transmission electron microscope (Hitachi, H9000NAR) equipped with a $\mathrm{LaB}_{6}$ electron source and an electron energy loss spectrometer (Gatan, 676 ). Spectra were recorded using a $300 \mathrm{keV}$ electron beam, and the energy resolution was deduced to be $2-3 \mathrm{eV}$ by referring the energy spread of the electron beam.

An empirical energy band was calculated by the tight-binding method ${ }^{18)}$ and the results were compared with the BIS, $\mathrm{XAS}$ and EELS spectra. Details of the calculation were reported elsewhere. ${ }^{15)}$

\section{Results}

Figure 1 shows BIS and PES spectra of $\mathrm{CaTiO}_{3}$. A total DOS by the energy band calculation is also shown in the figure. It is noted that there is no relationship between the BIS and PES spectra with respect to the relative intensities. The Fermi energy determined experimentally was set to zero in the energy scale in the two spectra, and the bottom of the conduction band was located at zero in the total DOS. An energy gap was observed between the valence band edge in the PES spectrum and the conduction band edge in the BIS spectrum, and the intensity of the BIS spectrum rises from the conduction band edge. This observation indicates that the Fermi energy lies at the bottom of the conduction band, and it is consistent with the fact that the sample is an $n$-type semiconductor due to Y-doping. ${ }^{16)}$ Two broad bands were observed at $\sim 2$ and $\sim 7 \mathrm{eV}$ above the Fermi energy in the BIS spectrum. Similar structure has been observed in the UV-BIS and X-ray-BIS spectra of $\mathrm{SrTiO}_{3}$ by Reihl et al. and Tezuka et al., ${ }^{6,7)}$ respectively.

Figure 2 shows the EELS and XAS spectra for (a) $\mathrm{Ca}$ $\mathrm{L}_{2,3}$, (b) $\mathrm{Ti} \mathrm{L}_{2,3}$, and (c) O K edges of $\mathrm{CaTiO}_{3}$. The XAS spectra are adjusted to the EELS spectra in the energy

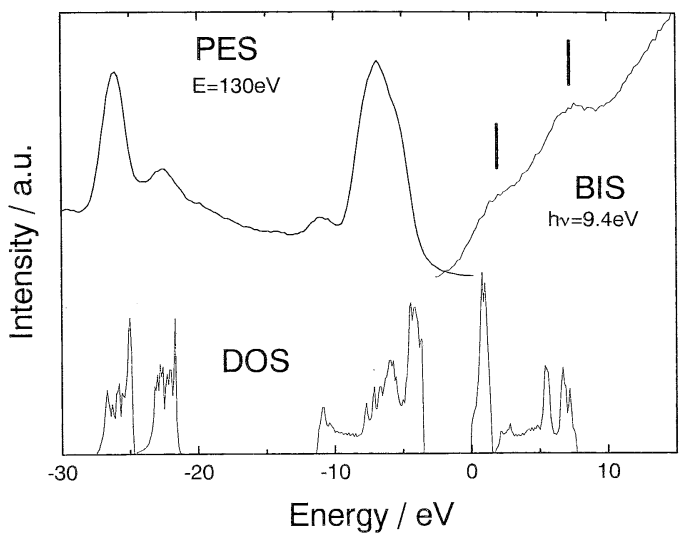

Fig. 1. BIS and PES spectra of $\mathrm{CaTiO}_{3}$ with DOS estimated by an energy band calculation.
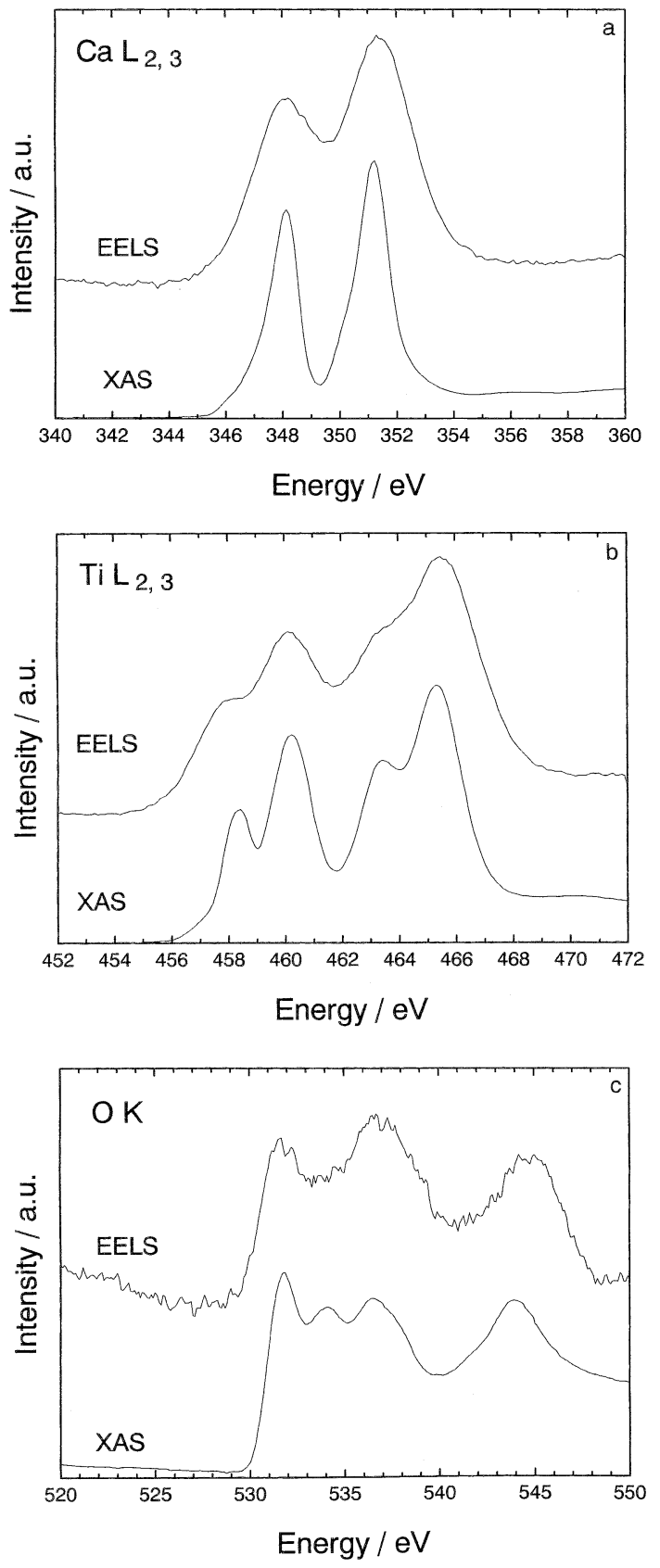

Fig. 2. EELS and XAS spectra of $\mathrm{Ca}_{2,3}$ (a), Ti $\mathrm{L}_{2,3}$ (b) and $\mathrm{OK}$ (c) edges for $\mathrm{CaTiO}_{3}$. 
scale so that the energy of peaks agrees between the spectra, and intensities of the spectra are arbitrarily arranged to compare them. The XAS and EELS spectra provided analogous shape in each of the three absorption edges, although the former gave sharper bands because of the higher energy resolution.

Two bands were observed at 348 and $351 \mathrm{eV}$ in the $\mathrm{Ca}$ $\mathrm{L}_{2,3}$ edges (Fig. 2(a)), and they are attributed to the $\mathrm{L}_{2}$ and $\mathrm{L}_{3}$ edges, respectively. The spectrum for $\mathrm{Ti} \mathrm{L}_{2,3}$ edges (Fig. 2(b)) is also interpreted in the similar manner, except that each unoccupied band is split into two bands under the influence of octahedral crystal fields. A pair of bands at 458 and $460 \mathrm{eV}$ is ascribed to the $\mathrm{L}_{2}$ edge, and the other one at 463 and $465 \mathrm{eV}$ is to the $\mathrm{L}_{3}$ edge. No prominent absorption for $\mathrm{Ca}$ and $\mathrm{Ti} \mathrm{L}_{1}$ edges was detected in the present measurement. The $\mathrm{O} \mathrm{K}$ edge (Fig. 2(c)) shows four bands at $531,534,536$ and $544 \mathrm{eV}$. Since the intensity of the spectra for the $\mathrm{OK}$ edge was lower than those for the $\mathrm{Ca} \mathrm{L}_{2,3}$ and $\mathrm{Ti} \mathrm{L}_{2,3}$ edges, the signal/noise ratio of the $\mathrm{O} \mathrm{K}$ edge spectra was relatively low, especially for the EELS spectrum. The energies of the absorption edges were approximately estimated from the lower-energy edge of the observed bands to be 346 and $349 \mathrm{eV}$ for the $\mathrm{CaL}_{2}, \mathrm{~L}_{3}$ edges, 456 and $461 \mathrm{eV}$ for the $\mathrm{TiL}_{2}, \mathrm{~L}_{3}$ edges and $530 \mathrm{eV}$ for the $\mathrm{O}$ $\mathrm{K}$ edge, which were close to the energies reported by Bearden and Burr. ${ }^{19)}$ The XAS spectra were used for the analysis of the conduction band in the following discussions because the XAS spectra gave sharper features than the EELS spectra.

\section{Discussion}

\subsection{Comparison of XAS spectra with PDOS}

XAS and EELS spectra generally provide the PDOSs of conduction bands, while BIS spectrum does the total DOS. The former two spectra originate mainly from intra-atomic excitations, and an absorption or energy loss in the spectra is characterized by each element in materials. Recalling the selection rule for electric dipole transitions in atoms,

$$
\Delta l= \pm 1, \Delta m=0, \pm 1
$$

where $l$ is an azimuthal quantum number and $m$ is a magnetic quantum number, transitions to s or d orbitals are allowed for $\mathrm{L}_{2,3}$ edges and transitions to $\mathrm{p}$ orbitals are permitted for a $\mathrm{K}$ edge. Therefore, it is expected that the $\mathrm{Ca}$ $\mathrm{L}_{2,3}$ edges show the transition to a $\mathrm{Ca} 4 \mathrm{~s}$ band, the $\mathrm{Ti} \mathrm{L}_{2,3}$ edges reflect the transition to $\mathrm{Ti} 3 \mathrm{~d}$ and $4 \mathrm{~s}$ bands, and the $\mathrm{O}$ $\mathrm{K}$ edge does the transition to an $\mathrm{O} 2 \mathrm{p}$ band in the anti-bonding states. Although the transition to a Ca $3 \mathrm{~d}$ band may be possible in the $\mathrm{Ca} \mathrm{L}_{2,3}$ edges, it was tentatively neglected in the present analysis because $\mathrm{Ca} 3 \mathrm{~d}$ orbitals are unoccupied even in $\mathrm{Ca}$ atoms and the determination of their energy was beyond the approximation of our present calculation. Provided that the band width of the core levels or initial states in each transition is negligibly small compared with that of the conduction band, the structure of the spectra is substantially determined by the structure of the final states, that is, the PDOSs of the conduction band for each element. On the basis of this assumption, the XAS spectra were compared with the results of an energy band calculation. In the comparison, the XAS spectra for $\mathrm{Ca}$ and $\mathrm{Ti}$ edges were modified because our calculation did not include the spin effect which causes the energy splitting of $\mathrm{L}_{2}$ and $\mathrm{L}_{3}$ edges. The modification was carried out to eliminate the $\mathrm{L}_{3}$ edges in the way that the high energy sides of the $\mathrm{L}_{2}$ edges were smoothly extrapolated to zero. It should be noted that the simple modification erases some absorptions at higher energy such as the absorption by a Ti 4 s band.

Figure 3 shows the comparison of the XAS spectra with the PDOSs obtained by the tight-binding energy band calculation. The bottom of the conduction band is set to

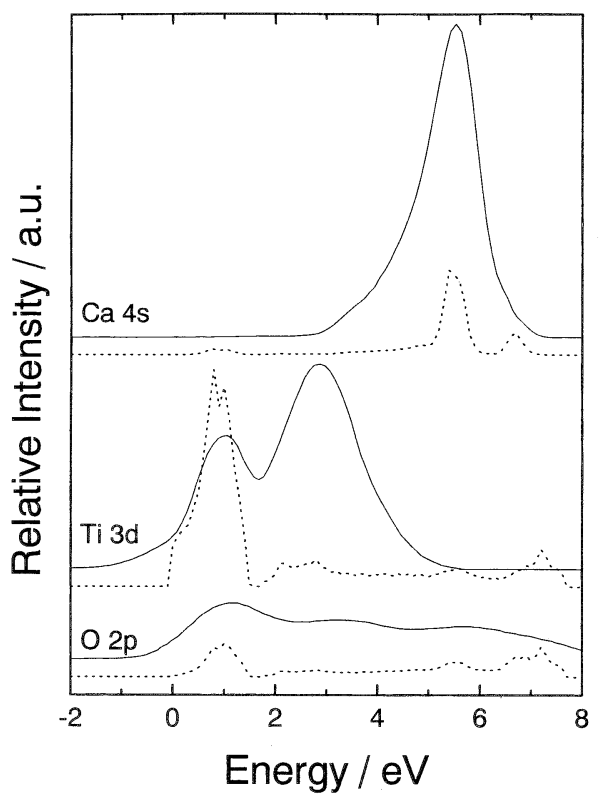

Fig. 3. Comparison of the XAS spectra (solid lines) for $\mathrm{Ca} \mathrm{L}_{2}$ (top), $\mathrm{Ti} \mathrm{L}_{2}$ (middle), and $\mathrm{O} \mathrm{K}$ (bottom) edges with the PDOSs (dotted lines) of $\mathrm{Ca} 4 \mathrm{~s}$, Ti $3 \mathrm{~d}$ and $\mathrm{O} 2 \mathrm{p}$ orbitals. The XAS spectra were adjusted in the energy scale to fit to the corresponding PDOSs.

zero in the energy scale for the PDOSs, as done in Fig. 1. The energy scale for the XAS spectra was taken so that the feature of the spectra fits to the respective PDOSs. Although the comparison may be somewhat artificial, it is based on the selection rule and provides some reasonable results: the degree of the energy split by crystal fields observed in Ti edges is almost the same between the XAS spectrum and PDOS, and the energies of three bands in the XAS spectra for the O K edge agree well with those of the bands for $\mathrm{Ca}$ and $\mathrm{Ti} \mathrm{L}_{2}$ edges indicating the anti-bonding states of $\mathrm{O} 2 \mathrm{p}$ bands. In spite of some deviations in the intensities between the XAS spectra and PDOSs due to the neglect of the transition probability, they agree with each other semi-quantitatively.

\subsection{Band assignment in BIS spectrum}

It is anticipated that the BIS spectrum can be in principle simulated by the sum of the XAS spectra, and it makes band assignments in the BIS spectrum persuasive. To carry out the simulation, it should be noted that the relative intensities in the XAS spectra are meaningful information about the PDOSs because they were measured sequentially under the same conditions and the energy dependence of the power of the light source was properly corrected by the yield current of Au. The XAS spectrum for each element in Fig. 3 was simply added together to synthesize a trial spectrum and the spectrum synthesized was compared with the BIS spectrum.

Figure 4 shows the BIS spectrum and the spectrum synthesized by the sum of the XAS spectra. The energy position of the XAS spectra in Fig. 4 is identical with that in Fig. 3. Some deviations were observed between the two spectra: a band split around $2 \mathrm{eV}$ in the synthesized XAS spectra was not observed in the BIS spectrum, and peaktop energies of bands do not perfectly agree between the spectra. It seems that the energy resolution and the sensitivity of the detector was not high enough to resolve the band split in the present BIS measurement. In addition, some errors in the empirical energy band calculation, by which the energy position of the XAS spectra was determined, might cause the mismatch of the peak-top energies. 


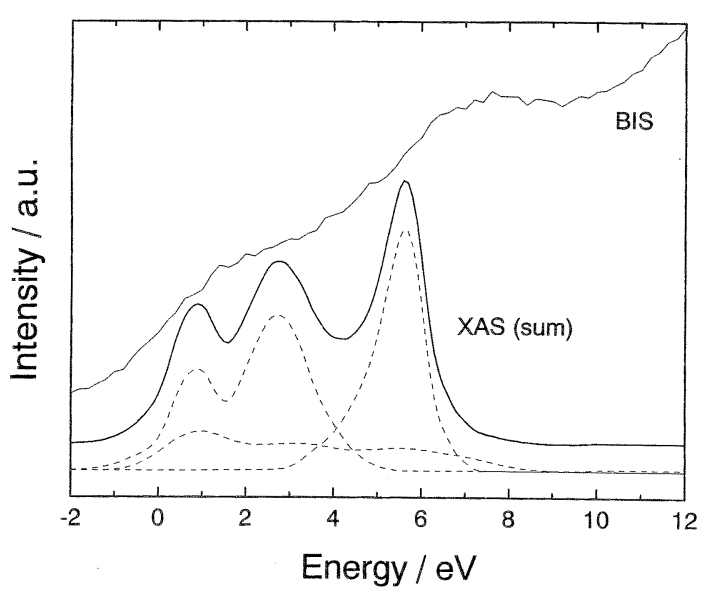

Fig. 4. Comparison of the BIS spectrum (solid line: top) with the spectrum (solid line: bottom) synthesized by the sum of the XAS spectra for each element (dashed lines).

However, in spite of the deviations, the spectrum synthesized agrees with the BIS spectrum on the whole, and it was concluded from the comparison that the two bands at 2 and $7 \mathrm{eV}$ in the BIS spectrum are related to a pair of $\mathrm{Ti} 3 \mathrm{~d}$ bands at 1 and $3 \mathrm{eV}$ and a $\mathrm{Ca} 4 \mathrm{~s}$ band at $6 \mathrm{eV}$ in the synthesized XAS spectrum, respectively.

The XAS spectrum for the $\mathrm{Ti} \mathrm{L}_{2,3}$ edges in $\mathrm{CaTiO}_{3}$ is analogous to that in $\mathrm{SrTiO}_{3}$ reported by Abbate et al. ${ }^{11)}$ Moreover, similar spectral features were reported in the UV-BIS and X-ray-BIS spectra of $\mathrm{SrTiO}_{3}$ by Reihl et al. ${ }^{6}$ and Tezuka et al, ${ }^{7)}$ This similarity probably originates from the analogous crystal structure between $\mathrm{CaTiO}_{3}$ and $\mathrm{SrTiO}_{3}$ even if $\mathrm{CaTiO}_{3}$ has orthorhombic distortion from an ideal cubic perovskite structure. As Reihl et al. mentioned, there may be some contribution of a Ti $4 \mathrm{~s}$ band to the structure of the conduction band, which was neglected in the present analysis of the XAS spectrum. However, no remarkable band originated from the $\mathrm{Ti}$ 4s band was observed in our XAS spectrum of $\mathrm{Ti} \mathrm{L}_{2,3}$ edges for $\mathrm{CaTiO}_{3}$ and even in the high-resolution XAS spectrum for $\mathrm{SrTiO}_{3}$ by $\mathrm{Ab}$ bate et al. This fact suggests that the contribution of the $\mathrm{Ti}$ $4 \mathrm{~s}$ band to the conduction band is fairly small in these alkaline earth titanates. Considering the small contribution of the Ti $4 \mathrm{~s}$ band to the conduction band, the intensity of the XAS spectrum for the $\mathrm{Ca}_{2,3}$ edges is rather high for the transition to the $\mathrm{Ca} 4 \mathrm{~s}$ band. This may suggest that the $\mathrm{Ca} 3 \mathrm{~d}$ band, which was neglected in the present analysis, is partially included in the conduction band. More accurate calculations will be necessary for further analysis of the electronic structure as well as experimental techniques with higher energy resolution.

\section{Conclusion}

The BIS, XAS and EELS spectra of $\mathrm{CaTiO}_{3}$ were measured to understand the structure of the conduction band. Two broad bands at 2 and $7 \mathrm{eV}$ were observed in the BIS spectrum. The XAS and EELS spectra for the absorption edges of identical elements gave almost the same features, and the energy resolution of the XAS spectra was higher than that of the EELS spectra in the present experiments. It was understood from the comparison of the XAS spectra with the calculated PDOSs that the conduction band mainly consists of $\mathrm{Ca} 4 \mathrm{~s}$ and $\mathrm{Ti} 3 \mathrm{~d}$ bands with a small contribution of the $\mathrm{O} 2 \mathrm{p}$ anti-bonding bands. A spectrum synthesized by the sum of the XAS spectra provides three bands at 1,3 and $6 \mathrm{eV}$, and the feature of the spectrum is similar to that of the BIS spectrum. From the comparison of the BIS spectrum with the XAS spectrum synthesized, it was concluded that the two bands at 1 and $3 \mathrm{eV}$ derived from the Ti $3 \mathrm{~d}$ bands in the XAS spectrum are responsible for the band at $2 \mathrm{eV}$ in the BIS spectrum, and the other band at $6 \mathrm{eV}$ related to the $\mathrm{Ca} 4 \mathrm{~s}$ band in the XAS spectrum is for the band at $7 \mathrm{eV}$ in the BIS spectrum.

References

1) S. Sasaki, C. T. Prewitt, J. D. Bass and W. A. Schulze, Acta Cryst., C43, 1668-74 (1987).

2) A. H. Kahn and A. J. Leyendecker, Phys. Rev., 135, A132125 (1964).

3) T. F. Soules, E. J. Kelly, D. M. Vaught and J. W. Richardson, Phys. Rev. B, 6, 1519-32 (1972).

4) L. F. Mattehiss, Phys. Rev. B, 6, 4718-40 (1972).

5) F. L. Battye, H. Höchst and A. Goldmann, Solid State Commun., 19, 269-71 (1976).

6) B. Reihl, J. G. Bednorz, K. A. Müller, Y. Jugnet, G. Landgren and J. F. Morar, Phys. Rev. B, 30, 803-06 (1984).

7) Y. Tezuka, S. Shin, T. Ishii, T. Ejima, S. Suzuki and S. Sato, J. Phys. Soc. Japan, 63, 347-57 (1994).

8) M. Cardona, Phys. Rev., 140, A651-55 (1965).

9) Y. Yacoby and O. Naveh, Phys. Rev. B, 7, 3991-4000 (1973).

10) D. Bäuerle, W. Braun, V. Saile, G. Sprüssel and E. E. Koch, Z. Physik, B29, 179-84 (1978).

11) M. Abbate, F. M. F. de Groot, J. C. Fuggle, A. Fujimori, Y. Tokura, Y. Fujishima, O. Strebel, M. Domke, G. Kaindl, M. Sacchi and N. Tsuda, Phys. Rev. B, 44, 5419-22 (1991).

12) P. Pertosa and F. M. Michel-Calendini, Phys. Rev. B, 17, 2011-20 (1978).

13) A. Frova and P. J. Boddy, Phys. Rev. Lett., 16, 688-90 (1966).

14) A. Frova and P. J. Boddy, Phys. Rev., 153, 606-16 (1967).

15) K. Ueda, H. Yanagi, H. Hosono and H. Kawazoe, submitted to Phys. Rev. B.

16) K. Ueda, H. Yanagi, H. Hosono and H. Kawazoe, Phys. Rev. $B, 56,12998-3005$ (1997).

17) H. Kawazoe, M. Yasukawa, H. Hyodo, M. Kurita, H. Yanagi and H. Hosono, Nature, 389, 939-42 (1997).

18) W. A. Harrison, "Electronic Structure and the Properties of Solids," W. H. Freeman and Company, San Francisco (1980) Chapter 4.

19) J. A. Bearden and A. F. Burr, Rev. Mod. Phys., 39, 125-42 (1967). 